\title{
SISTEMA DE INFORMAÇÃO GEOGRÁFICA COMO FERRAMENTA DE APOIO AO LICENCIAMENTO AMBIENTAL NO ESTADO DE GOIÁS BRASIL
}

\author{
HELBER DE CARVALHO
}

Mestre em Geografia. Técnico do Instituto Mauro Borges de Estatísticas e Estudos Socioeconômicos -IMB, Universidade Federal de Goiás, Campus Samambaia ${ }^{1}$

helbercarvalho10@gmail.com

\section{FABRIZIA GIOPPO NUNES}

Doutora em Geologia Ambiental. Docente do curso de Pós-graduação em Geografia Instituto de Estudos Socioambientais, Universidade Federal de Goiás, Campus Samambaia ${ }^{1}$ fabrizia.iesa.ufg@gmail.com

\section{ALEX MOTA DOS SANTOS}

Doutor em Geografia. Docente do curso de Mestrado Profissional em Tecnologia de Processos Sustentáveis do IFG, Professor do curso de Engenharia de Transporte Universidade Federal de Goiás, Campus Aparecida de Goiânia ${ }^{2}$ alex.geotecnologias@gmail.com

\begin{abstract}
RESUMO: A capacidade de um Sistema de Informação Geográfica (SIG) no tratamento de dados espaciais possibilita armazenar, analisar e disseminar informações com a potencialidade de executar análises complexas, fator importantíssimo ao licenciamento ambiental. Nesse sentido, o presente trabalho objetivou diagnosticar quais benefícios são desencadeados, quando adotadas ferramentas de SIG, nos procedimentos de Licenciamento Ambiental do Estado de Goiás. Para tanto, foram avaliadas as legislações ambientais, realizadas pesquisas bibliográficas e consultas a experiências anteriores. Foi averiguado também, o atual estágio de uso dessa tecnologia no órgão ambiental responsável (SECIMA), e efetuadas avaliações das rotinas de licenciamento. Além de entrevistas com técnicos e gestores, estabelecendo quais seriam os principais requisitos necessários para estimular as práticas em geotecnologias. O resultado do trabalho permitiu a criação de um protótipo de SIG online - Mapa Interativo $W e b$, possibilitando o licenciamento ambiental melhor embasado, pelas práticas das geotecnologias.
\end{abstract}

Palavras-chave: Geotecnologias; licenciamento ambiental; mapa interativo; WebGIS.

\section{GEOGRAPHIC INFORMATION SYSTEM AS A TOOL TO SUPPORT ENVIRONMENTAL LICENSING IN THE STATE OF GOIÁS BRAZIL}

ABSTRACT: The ability of a Geographic Information System (GIS) in data processing makes it possible to store, analyze and disseminate information with the potential to perform complex analyzes, a very important factor in environmental licensing. In this context, the present proposal seeks to verify which benefits are triggered by GIS instruments, in the Environmental Licensing procedures of the state of Goiás. Environmental legislation has been evaluated, bibliographic research and previous experiences have been carried out. It was also verified, the current stage of use of GIS in the responsible environmental agency (SECIMA), and evaluations were carried out in the licensing routines. As well as interviews with technicians and managers, establishing what would be the main requirements needed to meet the main practices in geoprocessing. The result of the work allowed the creation of an Interactive Map - GIS online, enabling a better environmental licensing, by the practices of geotechnologies.

Keywords: Geotechnologies; environmental licensing; interactive map; WebGIS.

\footnotetext{
${ }^{1}$ Endereço para correspondência: Avenida Esperança, s/n - Campus Samambaia, CEP: 74001-970, Goiânia, Goiás - Brasil.

${ }^{2}$ Endereço para correspondência: Rua Mucuri, s/n - Setor Conde dos Arcos, CEP: 74968755, Aparecida de Goiânia, Goiás - Brasil.
}

Helber de Carvalho, Fabrizia G. Nunes, Alex M. dos Santos. Sistema de informação geográfica como ferramenta de apoio ao (...) Brazilian Geographical Journal: Geosciences and Humanities research medium, Ituiutaba, v. 11, n. 2, p. 4-21, ago./dez. 2020 


\title{
SISTEMA DE INFORMACIÓN GEOGRÁFICA COMO HERRAMIENTA DE APOYO A LAS
} LICENCIAS AMBIENTALES EN EL ESTADO DO GOIÁS BRASIL

\begin{abstract}
RESUMEN: La capacidad de un Sistema de Información Geográfica (SIG) en el tratamiento de datos espaciales permite almacenar, analizar y difundir información con el potencial de realizar análisis complejos, un factor muy importante en las licencias ambientales. En este sentido, el presente estudio tuvo como objetivo diagnosticar qué beneficios se desencadenan, al adoptar instrumentos de SIG, en los procedimientos de Licenciamiento Ambiental del Estado de Goiás, para lo cual se evaluaron las leyes ambientales, se realizaron investigaciones bibliográficas y consultas con experiencias previas. También se investigó la etapa actual de uso de SIG en la agencia ambiental responsable (SECIMA) y se llevaron a cabo evaluaciones en las rutinas de concesión de licencias. Además de entrevistas con técnicos y gerentes, establecer cuáles serían los principales requisitos necesarios para estimular las prácticas de geotecnologías. El resultado del trabajo permitió la creación de un prototipo de SIG en línea - Mapa Interactivo Web, que permite una mejor licencia ambiental, basada en las prácticas em geoprocesamiento.
\end{abstract}

Palabras clave: Geotecnologías; licenciamiento ambiental; mapa interactivo; WebGIS.

\section{INTRODUÇÃO}

Os Estudos e Relatórios de Impactos Ambientais (EIA/RIMA) constituem-se nos principais componentes para os gestores e analistas ambientais atestarem à viabilidade, ou não, de um determinado empreendimento e suas atividades. São documentos obrigatórios à avaliação técnica das solicitações de licenciamento, os quais serão decisivos para a maximização dos princípios de conservação e sustentabilidade ambiental.

Entretanto, no cenário nacional, diversos levantamentos concernentes à avaliação da qualidade da produção dos Estudos e Relatórios de Impactos Ambientais, indicam uma série de deficiências, relacionadas à condição e qualidade de seus dados. Um dos principais pontos constatados é a incompatibilidade técnica dos documentos fornecidos, ocasionando distorções na avaliação da viabilidade, ou não, do empreendimento. Tal inadequação influencia tanto nos aspectos técnicos, como na definição inadequada de medidas de mitigação e compensação ambiental, quanto em elementos relacionados à rotina administrativa, constatada na morosidade e pendências de ajustes dos processos para a continuidade da avaliação.

Outro elemento importante a ser mencionado, diz respeito a insuficiência de dados e informações. A carência de informações é um grave entrave para a correta verificação da avaliação ambiental dos empreendimentos e de suas atividades, podendo resultar em distorções, não somente no escopo do licenciamento, mais também ocasionando uma conjuntura de desdobramentos que afetam outros componentes da administração pública. Essa insuficiência pode ser oriunda tanto da ausência de dados fundamentais que deveriam estar presentes nos estudos e relatórios elaborados pelos responsáveis técnicos, quanto na inadequação do sistema do próprio órgão ambiental, que não dispõe de um aparato de recursos que proporcione abrigar, gerenciar, disseminar e integrar com eficiência e transparência os dados concernentes à questão ambiental.

Na tentativa de amenizar essa situação, diversos são os autores que têm apresentado propostas para a apreensão e fortalecimento do licenciamento ambiental no Brasil (BARRETO, 2012; PAES, 2010; SAITO, 2010 e DA ROCHA, 2014). Dentre suas propostas, nota-se a concentração de esforços para a reunião e sistematização de informações coerentes ao licenciamento ambiental, bem como, a intenção da adoção de uma estrutura ambiental consolidada, salientando esforços para a criação de uma base integrada. Nesse contexto, é sugerida a implantação de um banco de dados integrado a uma rede nacional de informações ambientais, subsidiando as ações que se fizerem necessárias.

Como referência de apoio podemos citar o trabalho de Nascimento (2011), que desenvolveu um Sistema de Informações Ambientais aplicado ao licenciamento ambiental do Estado de São Paulo. Além deste, Saito (2010) destaca os aspectos jurídicos, apresentando um diagnóstico dos principais entraves no cenário do licenciamento ambiental, com ênfase, nas competências do sistema brasileiro. Já no trabalho de Paes (2010) é abordada a contribuição da Ciência Geográfica 
ao licenciamento ambiental e ao estudo de impacto ambiental. Nesta perspectiva, Barreto (2012) estabelece, por meio de análises, a delimitação do escopo em processos de avaliação de impactos ambientais. Especificamente para a implementação de uma plataforma de Sistema de Informação Geográfica (SIG), o trabalho de Da Rocha (2014) fornece vasta referência bibliográfica sobre mapas interativos, além de descrever minuciosamente a criação de um SIG online.

É importante destacar, que na análise ambiental são imprescindíveis os aspectos geográficos e o acesso as informações dos atributos descritivos das áreas a serem avaliadas, além dos meios técnicos-científicos para analisar e integrar dados distintos. Nesse cenário, assim como em todo universo da Geoinformação, já é de conhecimento que qualquer informação da superfície terrestre pode ser modelada em um Sistema de Informação Geográfica (SIG). Essa potencialidade vincula a feição real com a sua representação espacial (FAVRIN, 2009).

Dessa forma, a capacidade do SIG no tratamento de dados geográficos estende-se, desde o armazenamento da geometria do perímetro do empreendimento a ser licenciado, a potencialidade de executar análises complexas de diversas fontes, criando um banco de dados georreferenciado (CÂMARA; DAVIS, 2004). Nestes termos, para Estes; Star (1990), os recursos do SIG envolvem a capacidade de reunir e avaliar diversas informações espaciais.

A adoção destas ferramentas computacionais na representação espacial, reduz o tempo da geração dos dados, potencializando a disseminação da ciência cartográfica, cada vez mais presente em diversas áreas do conhecimento. Neste universo, surge a internet com a importante função de disseminação dos conceitos cartográficos em diversos formatos e, atingindo inúmeros públicosalvo. Como exemplo, temos o WebGIS - termo que se refere as plataformas que integram dados espaciais online. Com esta tecnologia torna-se possível visualizar planos de informações geográficas e efetuar consultas que antes eram inacessíveis nos mapas tradicionais.

Essa estruturação oferece um gerenciamento dos dados mais apurado, acarretando em uma interpretação mais precisa do mundo real. Nesse sentido, o fomento a esses instrumentos é de extrema relevância para o entendimento dos processos ambientais, propiciando um direcionamento mais ajustado das ações para preservação e conservação dos recursos naturais.

Vale lembrar, que em um país de dimensão continental, o uso das ferramentas de geoprocessamento, como os SIGs é essencial para seu entendimento (CÂMARA et al., 2000). Já é notório, que nos últimos anos, diversos setores da administração pública nacional obtiveram crescimento no uso de geotecnologias. Entretanto, essa expansão não atingiu sua plenitude na maioria dos órgãos públicos brasileiros relacionados ao meio ambiente. No cenário goiano, na competência estadual, estudos revelam entraves no licenciamento ambiental, devido à ausência de dados geoespaciais. Assim, de acordo com a pesquisa de Carvalho et al. (2013), observa-se a necessidade do aperfeiçoamento e disseminação de novas alternativas das geotecnologias nas rotinas de trabalho dos técnicos e analistas da Secretaria Estadual do Meio Ambiente de Goiás (SECIMA).

Diante do exposto e direcionado a tal problemática, este trabalho objetivou a implementação de um banco de dados espaciais, integrado a uma rede nacional, proporcionando um dispositivo para nortear as ações de licenciamento ambiental no Estado de Goiás. Para tanto, avaliou-se os efeitos da incorporação de novas alternativas tecnológicas para a análise da dimensão geográfica dos licenciamentos ambientais, a cargo do órgão ambiental competente, foco dessa pesquisa. Assim, além de diagnosticar as atuais práticas das geotecnologias no órgão em questão, buscou também sondar e implementar propostas que incorporem efetivamente meios de apoio das ferramentas de SIG, em suas diversas rotinas de trabalho.

\section{INSTRUMENTOS DO LICENCIAMENTO AMBIENTAL}

O licenciamento ambiental consiste em um mecanismo de planejamento e gestão ambiental, instituído pela Política Nacional do Meio Ambiente (BRASIL, 1986). Visa analisar a viabilidade do empreendimento, considerando a revisão de procedimentos e critérios que garantam ações compatíveis com os princípios do desenvolvimento sustentável e que promovam a melhoria contínua.

Helber de Carvalho, Fabrizia G. Nunes, Alex M. dos Santos. Sistema de informação geográfica como ferramenta de apoio ao (...) Brazilian Geographical Journal: Geosciences and Humanities research medium, Ituiutaba, v. 11, n. 2, p. 4-21, ago./dez. 2020 
Para sua real efetivação o órgão ambiental competente define as condições, restrições e meios de gerenciamento ambiental que deverão ser obedecidos pelo empreendedor por meio de ato administrativo. Esse gerenciamento contempla as pessoas físicas e jurídicas, estabelecendo normas para localizar, instalar, ampliar e operar atividades ou empreendimentos sujeitos ao licenciamento. Essas normativas pretendem certificar a ação governamental e têm como objetivo disciplinar e regulamentar o acesso aos recursos naturais, com a inclusão de mecanismos, que harmonizem as atividades econômicas, prevendo medidas de preservação dos recursos naturais e/ou de mitigação de prováveis danos ambientais (SÁNCHEZ, 2013).

Dentre os instrumentos presentes nos trâmites processuais do licenciamento destaca-se os Estudos e Relatórios Ambientais (CÂMARA; MEDEIROS, 1998). Esses documentos compreendem todos e quaisquer levantamentos relativos aos aspectos ambientais concernentes ao posicionamento geográfico, implantação, execução e ampliação de uma atividade ou empreendimento, subsidiando a avaliação da licença requerida. Os documentos buscam o diagnóstico da solicitação, possuindo diversos formatos, tais como: o Estudo de Impacto Ambiental (EIA), acompanhado de seu respectivo Relatório de Impacto Ambiental (RIMA), plano e relatório ambiental, projeto de controle ambiental, diagnóstico ambiental, plano de manejo, plano de recuperação de área degradada e análise preliminar de risco.

A Avaliação de Impacto Ambiental (AIA) apoia-se nos elementos técnicos disponibilizados nos Estudos Ambientais para nortear medidas que certifiquem a avaliação de determinado projeto, propenso ou com características passíveis à geração de danos ambientais e, que englobe ações prevendo a magnitude e a intensidade dos possíveis impactos. Além de avaliar os prováveis impactos, que serão ocasionados pelo empreendimento, propõe alternativas mitigadoras. Desse modo, a AIA é um instrumento da política ambiental potencialmente eficaz na prevenção de danos ambientais, com o intuito da promoção do desenvolvimento sustentável.

Com o advento da AIA houve um estímulo à inserção da participação do público interessado nas etapas preliminares do licenciamento. Diante dessa alternativa, verifica-se o fortalecimento dos mecanismos que aprimoram a qualidade dos pareceres emitidos, ressaltando o papel essencial da participação da sociedade. Para respaldar esse processo, o licenciamento ambiental revela-se como um mecanismo de interação coletiva, ampliando a comunicação com a sociedade, ofertando múltiplas maneiras de interagir e colaborar com o processo.

Dessa forma, por meio de uma análise crítica das ações voltas à avaliação de impactos ambientais no cenário nacional, observa-se a necessidade de incrementar às bases de registros, informações e dados ambientais. Para tanto, sugere-se a implementação de um banco de dados ambientais integrado a uma rede nacional, proporcionando um dispositivo para nortear as ações de política e gestão do meio ambiente.

Assim, essas análises deveriam ocorrer com o aparato técnico e estratégico que englobasse os diversos temas e interações presentes no meio ambiente. Em virtude da complexidade desta questão, torna-se indispensável uma avaliação multifacetada que permita, além da integração de fatores distintos, como aspectos econômicos, sociais e ambientais, a própria inter-relação entre eles. Para tanto, é requerida a união de diferentes áreas e agentes do conhecimento científico para tratar do assunto. Essa característica traz a luz a pluralidade da questão: onde "os estudos ambientais além do caráter interdisciplinar são interdependentes, portanto devem ser apreciados conjuntamente" (NASCIMENTO; MONTAÑO, 2011).

Neste aspecto podemos citar o estudo de Oliveira et al. (2008), quando os autores apresentam as técnicas de geoprocessamento como ferramentas essenciais ao licenciamento ambiental de postos de combustíveis, demostrando as várias maneiras que a utilização das ferramentas de geotecnologias pode auxiliar no controle, monitoramento e tomada de decisão. Outro estudo a ser destacado é o trabalho da Embrapa Informática Agropecuária, desenvolvido por Speranza e Silva (2010), na elaboração de uma ferramenta computacional para auxiliar à análise técnica de processos de Licenciamento Ambiental, mediante a implementação das geotecnologias no Instituto de Meio Ambiente do Estado do Mato Grosso do Sul (IMASUL).

Como experiências internacionais ressaltamos o trabalho desenvolvido por Zheng; Chang; Fei (2017), do Departamento de Planejamento, Instituto Especial de Desing e Pesquisa de

Helber de Carvalho, Fabrizia G. Nunes, Alex M. dos Santos. Sistema de informação geográfica como ferramenta de apoio ao (...) Brazilian Geographical Journal: Geosciences and Humanities research medium, Ituiutaba, v. 11, n. 2, p. 4-21, ago./dez. 2020 
Engenharia Civil de Pequim - China, na simulação de planejamentos de tomada de decisão em WebGIS, como critério de avaliação dos impactos ambientais, para instalações de redes de transportes. Além deste, mencionamos também o trabalho de Vatresia; Sadler; Rais (2017) de gerenciamento ambiental com o uso de aplicativos da WebGIS, rumo à conservação da biodiversidade do Arquipélago da Indonésia, via sistema cibernético. Também a iniciativa de Tang et al. (2019) na criação de uma plataforma de WebGIS, direcionada à valorização da subsistência ambiental, frente ao desenvolvimento industrial no nordeste da Colúmbia Britânica.

\section{MATERIAIS E MÉTODOS}

O recorte espacial é o Estado de Goiás, no qual a SECIMA é o órgão responsável pelo licenciamento ambiental da maior parte de seus municípios. Para a elaboração do trabalho foram definidas 7 (sete) fases. A figura 1 apresenta o organograma da estrutura metodológica adotada, subdividido nas diversas fases norteadoras desta proposta.

Figura 1 - Organograma das fases metodológicas

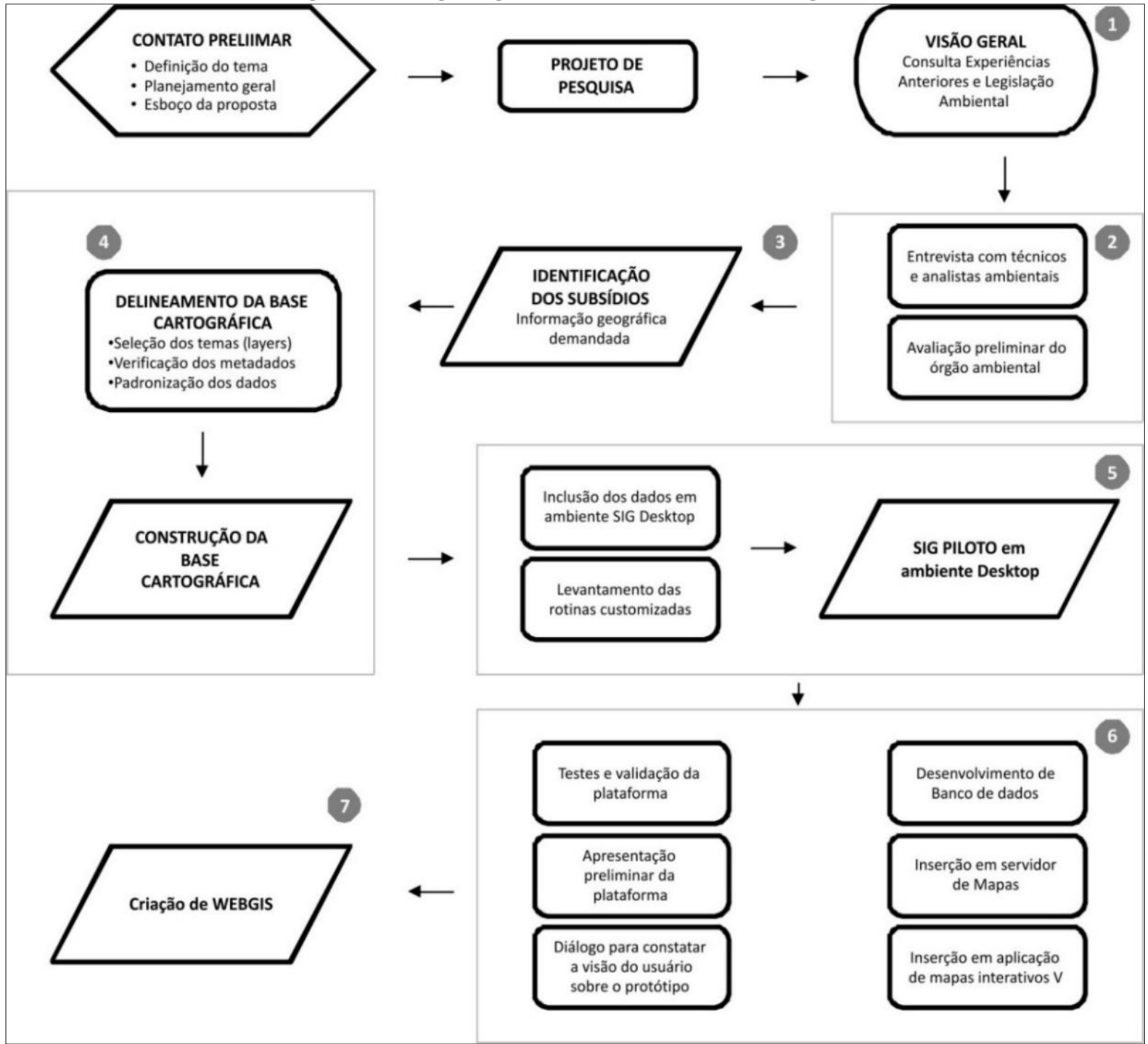

Elaborado pelos autores.

Helber de Carvalho, Fabrizia G. Nunes, Alex M. dos Santos. Sistema de informação geográfica como ferramenta de apoio ao (...) Brazilian Geographical Journal: Geosciences and Humanities research medium, 
Como ilustrado na figura acima, inicialmente realizou-se a revisão bibliográfica com o intuito de melhor compreender o funcionamento do licenciamento ambiental e suas relações com o universo geográfico, sobretudo no que concerne ao Sistema de Informação Geográfica e suas possibilidades de análise. Um dos aspectos importantes dessa fase foi à consulta a autores que tratam do assunto em questão, e que ofereceram suporte metodológico ao tema.

Dentre os autores consultados buscou-se compilar trabalhos que tratassem do aspecto jurídico do licenciamento ambiental, da avaliação de impactos ambientais, da definição da competência dos entes federativos e estaduais e, dos subsídios das geotecnologias como instrumento de apoio a análise de licenciamento e monitoramento ambiental. As experiências das práticas de SIGs onlines em outros órgãos ambientais foram pesquisadas, tanto no âmbito nacional, quanto regional. Assim, buscou-se identificar plataformas online que disponibilizem informações geográficas para o licenciamento ambiental e que utilizem mapas interativos. Também foram consultadas iniciativas voltadas para a disseminação de dados ambientais.

A segunda fase concentrou-se na consulta das rotinas processuais de análise da SECIMA, aprofundando, sobretudo, no estágio de utilização das geotecnologias. Para realizar essa etapa, optou-se por levantamentos empíricos, efetuados por meio de entrevistas semiestruturadas, conforme proposta de Melchiors e Medeiros (2017), na elaboração de um questionário e de observações feitas in loco dos processos constituintes do licenciamento ambiental.

Nessa etapa, foi efetuado um paralelo entre os requisitos previstos nas legislações ambientais, averiguados na etapa anterior, com o uso prático na Secretaria, sobretudo, nas avaliações que são efetuadas com o apoio do geoprocessamento e da aquisição de dados por geotecnologias.

Desta forma, foram efetuadas avaliações entre a rotina do licenciamento na SECIMA, as potencialidades da geotecnologia e a legislação ambiental pertinente para cada tipologia de licenciamento. Como exemplo, na tipologia de desmatamento, a legislação ambiental aponta determinados requisitos previstos no Novo Código Florestal como a proteção da mata ciliar, a manutenção da Reserva Legal e a realização da supressão arbórea, que deverá ocorrer somente com o Licenciamento Ambiental. Assim, verificou-se quais seriam as contribuições das geotecnologias para aquisição de dados e do SIG, para a integração destes, no diagnóstico da requisição do licenciamento para a supressão arbórea.

Com a obtenção das demandas de cada segmento usuário, levantadas no estágio anterior, na terceira fase realizou-se o processo de compilação dos dados necessários para compor o sistema. Essa etapa considerou as necessidades detectadas na fase anterior. Dessa maneira, foram compilados dados utilizados por técnicos e analistas ambientais (armazenados de forma pontuais nos computadores utilizados pelos servidores ambientais) e de dados provenientes de bases externas.

Ademais, foi efetuado um levantamento para verificar quais instituições públicas possuem bases cartográficas com temáticas relacionadas ao sistema, e que pudessem melhorar o banco de dados da SECIMA. Desse modo, o maior contingente de dados compilados veio de fontes externas ao órgão ambiental. Dentre as instituições fornecedoras destacam-se o Sistema Estadual de Geoinformação de Goiás (SIEG), o Laboratório de Processamento de Imagens e Geoprocessamento (LAPIG), a Plataforma do Ministério do Meio Ambiente (MMA), o United States Geological Survey (USGS), entre outros.

$\mathrm{Na}$ quarta fase realizou-se a integração dos dados utilizados no estágio anterior e a construção de uma Base Cartográfica, que atendesse as principais demandas, levantadas pelos gestores ambientais. Para a construção da Base Cartográfica foram armazenadas diversas temáticas, tais como: (a) imagens de satélites de diversas datas e, com recobrimento de todo o estado; (b) criação de MDT (Modelo Digital do Terreno) produzido com dados dos sensores ASTER e SRTM; (c) malha viária das principais rodovias de Goiás; (d) pedologia; (e) geomorfologia; (f) desmatamentos; (g) cobertura e uso do solo; (h) Unidades de Conservação Estaduais e Federais; (i) drenagens; entre outras.

A quinta fase destinou-se a elaboração de rotinas e métodos que potencializassem os procedimentos do licenciamento, direcionados principalmente as análises que necessitam de

Helber de Carvalho, Fabrizia G. Nunes, Alex M. dos Santos. Sistema de informação geográfica como ferramenta de apoio ao (...) Brazilian Geographical Journal: Geosciences and Humanities research medium, Ituiutaba, v. 11, n. 2, p. 4-21, ago./dez. 2020 
ferramentas de geoprocessamento. Gerando soluções customizadas a realidade do setor, essa fase buscou constatar quais seriam as aplicações, funcionalidades e potencialidades dos dados geográficos nas rotinas do licenciamento.

Foram consultados técnicos e gestores de diversas áreas, sondando as principais aplicações. O contato com os servidores constituiu em um elemento fundamental para conhecer quais expectativas foram geradas após o contato inicial (entrevista). Esse contato permitiu além de verificar os pontos fortes da proposta, o levantamento de pontos de aperfeiçoamentos no sistema, que não foram dimensionados na arquitetura inicial. Para efetuar esse processo foi realizado diálogos com os possíveis usuários do sistema (analistas ambientais, técnicos do órgão, responsáveis técnicos e agentes da sociedade), por meio da experiência de sua utilização, em um notebook com a Base Cartográfica inserida.

$\mathrm{Na}$ sexta fase, gerou-se o protótipo do SIG, objetivando englobar todas as bases cartográficas que darão suporte ao licenciamento ambiental. Para a sua construção optou-se por utilizar softwares livres como plataforma de SIG Desktop, com a intenção de sanar parcialmente as carências de dados de Geoinformação, sem acarretar custo financeiro para sua aquisição. Também foram aferidos os roteiros e técnicas a serem implantados junto aos técnicos e gestores envolvidos na Secretaria. O balanço desse processo serviu para mensurar a eficiência e eficácia do método e constatar suas prováveis adequações. Assim, esse feedback foi indispensável para constatar se o sistema atenderá as devidas exigências demandadas pelas análises ambientais, além da identificação de prováveis aperfeiçoamentos, assinalados pelos seus futuros usuários.

Por fim, a última fase, consistiu na elaboração da plataforma de WebGIS direcionada ao apoio das atividades de licenciamento ambiental da SECIMA. Para tanto, foram adotados softwares livres, tanto para a inserção de dados geográficos no servidor de mapas como o QGIS 3.6, quanto para a criação dos mapas interativos em uma única interface amigável da web como o software Leaflet.

Para a seleção dos programas bases, utilizados na arquitetura do WebGIS (mapa interativo), foram consultadas suas referências nas literaturas, além de pesquisas na internet. Os aspectos fundamentais para seleção dos softwares foram: (i) possibilitar o uso de funções de navegação, (zoom, pan e zoom extend); (ii) "resentar" de forma dinâmica os atributos tabulares; (iii) possibilitar a sobreposição de camadas de informações (overlay); (iv) ter compatibilidade com bases colaborativas (Google Maps, Bing, OpenStreetMap e outras); (v) compatibilidade para assimilar os padrões de extensão mais utilizados na representação geográfica, nos tipos vetoriais e matriciais (shapefile, Kml, GeoTIFF etc.); (vi) permitir a realização de consultas espaciais e; (vii) ter potencial para expansão de potencialidades como a inclusão de plugins.

Para compor as funcionalidades do mapa interativo, como já mencionado adotou-se o software Leaflet. Sua seleção decorreu da poderosa capacidade de agregar bibliotecas javascript, incluindo funcionalidades que podem ser inseridas no mapa principal. Após a visão geral das funcionalidades e características da ferramenta, ocorreu à compilação dos temas que atendam a maior parte das demandas constatadas via questionário e nas visitas feitas in loco, para avaliar as diversas atividades do licenciamento ambiental da SECIMA.

Dessa forma, os dados foram inseridos no software QGIS, para manipulação prévia dos arquivos vetoriais e matriciais. Foram definidas diversas simbologias que se mantiveram inalteradas, quando o arquivo no órgão de origem, permitia a identificação do estilo. Quando não foi possível utilizar o mesmo estilo (cor, espessura da linha, entre outros), em virtude da inacessibilidade da informação, foram adotadas características semelhantes.

Algumas edições de arquivos foram realizadas para atender a finalidade do projeto. Como exemplo, daqueles arquivos que apresentavam abrangência nacional. Nesses casos, ocorreu a adequação para o recorte espacial da área de interesse (Estado de Goiás) adotando como Sistema de Referência Geodésico o SIRGAS 2000 e mantendo uma escala compatível com a escala da fonte original, indicada nos próprios arquivos. Após a edição e definição da simbologia, os arquivos e projetos (qgs) foram introduzidos no QGIS Server. A escolha do QGIS Server como servidor de mapas, foi decorrente, principalmente, da compatibilidade entre o software Desktop (QGIS) e o servidor de mapas. 


\section{RESULTADOS E DISCUSSÕES}

Um dos primeiros aspectos observados no diagnóstico efetuado por intermédio da análise do questionário aplicado aos técnicos e gestores foi a necessidade da ampliação de medidas, que contemplem, com maior ênfase, o uso das geotecnologias para aquisição de dados e dos SIGs para sua integração em ambiente computacional. A inexistência de um setor específico que trate de geoprocessamento e a baixa regularidade de cursos fornecidos na área, além da aparente dificuldade do órgão ambiental em fomentar o desenvolvimento das práticas de geoprocessamento, compromete as avaliações ambientais.

$\mathrm{Na}$ estrutura dos dados, verificou-se que não existem mecanismos rigorosos de controle e especificações aos produtos cartográficos fornecidos, para a averiguação do Licenciamento Ambiental. Esse aspecto, afeta toda a cadeia de ações para a criação de um Banco de Dados Geográfico, desde o armazenamento até a disseminação das informações. Essa percepção está evidenciada nas respostas do questionário, representadas no gráfico da figura 2. Na citada figura é possível observar a existência de forte relação entre os itens referentes a necessidade de implantação de um sistema de armazenamento, integração e disseminação dos dados cartográficos.

Figura 2 - Resultados das entrevistas com técnicos e analistas ambientais

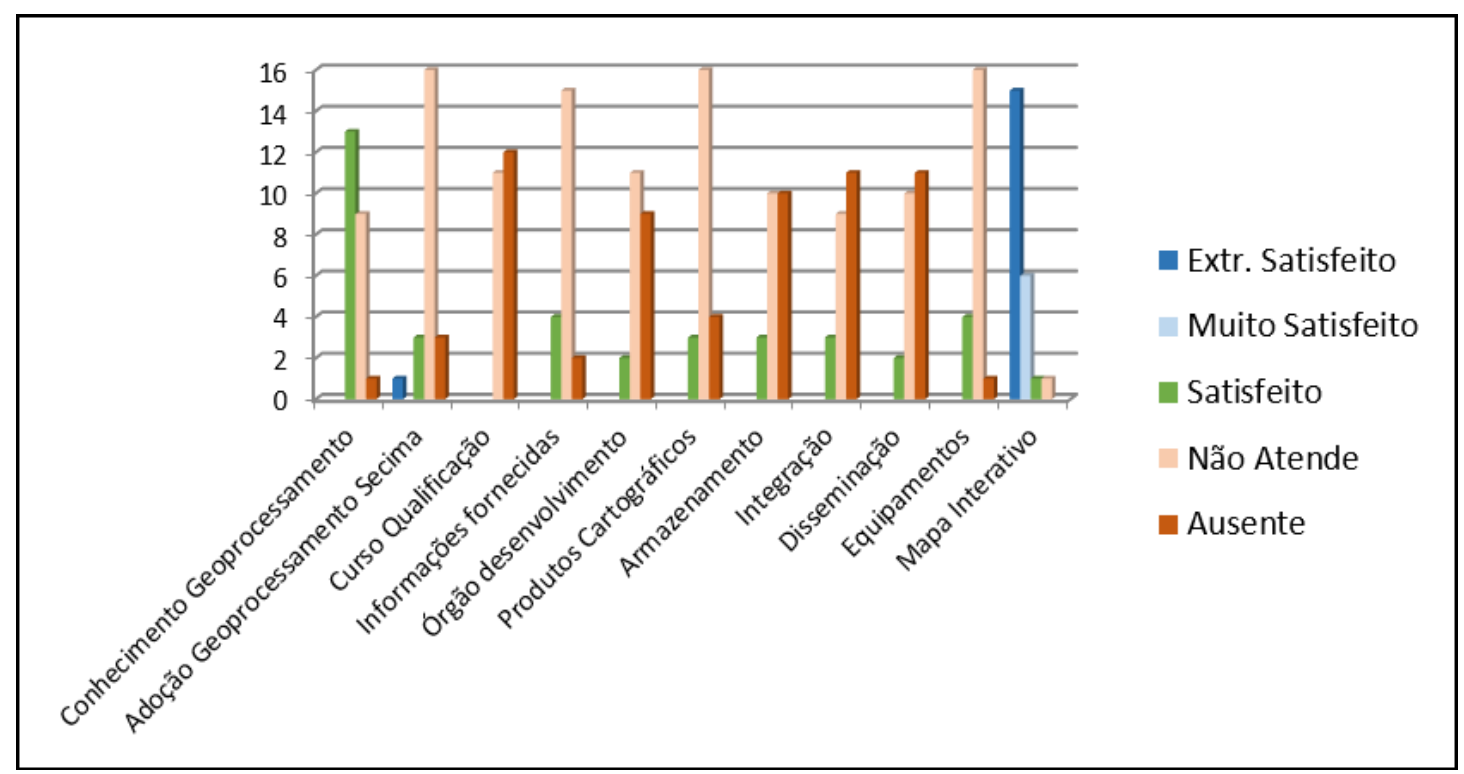

Elaborado pelos autores.

Outro aspecto importante a ser destacado é a provável compreensão distinta da semântica dos termos e conceitos inclusos no questionário. No item que trata do fornecimento das informações geográficas disponibilizadas pelos pleiteantes do licenciamento ambiental, verificou-se que 2 gestores descreveram a disponibilização como ausente. Entretanto, ressaltamos que a entrega dos produtos cartográficos, vinculados aos EIA/RIMA ocorre predominantemente nos formatos analógicos (croquis, plantas e mapas impressos) e em diversos formatos digitais (pdf, $\mathrm{dxf}, \mathrm{kml}$ ) entre outros.

Em todas as tipologias dos processos verificados foram identificadas a localização geográfica. Dessa forma, considera-se que os gestores que descreveram a disponibilização como ausente, interpretaram que os produtos não poderiam ser enquadrados como elementos cartográficos. Situação semelhante ocorreu com o item que trata do armazenamento das informações geográficas. Um volume muito expressivo, 10 gestores, responderam como 
ausente o processo de armazenamento. Não obstante, existi o armazenamento dos processos em um arquivo físico, incluindo os documentos, inclusive os cartográficos em CD's, quando disponibilizados em meio digital.

Tanto na existência da mídia digital (CD) como em arquivos físicos, os produtos cartográficos veem anexados ao processo, sendo de incumbência do técnico responsável a requisição. Até o presente momento não existia nenhum recurso que possibilitasse a inclusão em meio digital, compartilhado, dos produtos cartográficos para todos os gestores da SECIMA. Dessa forma, cada gestor é responsável em compilar sua coleção de dados espaciais em seu computador de uso particular, tornando o armazenamento pontual, sem a disseminação e integração dos dados com os demais técnicos.

Para a interpretação do uso do SIG na SECIMA foi definida a categorização numérica dos processos de licenciamento, por tipologias. Esse levantamento possibilitou constar quais são as tipologias de processos mais recorrentes. Como resposta foi efetuada uma consulta no sistema intranet, que contabilizou todas as requisições de licenciamentos cadastradas, entre os anos de 2008 até 2017. Essa categorização foi necessária devido ao volume de tipologias diagnosticadas, uma vez, que o levantamento inicial demonstrou a existência de 226 tipologias, com requisições distintas, nesse período. Tal diversidade de processos não permitiria a avaliação particular de cada tipologia. Entretanto, observou-se que $66 \%$ das requisições de licenciamento presentes no período, encontram-se nas 20 tipologias mais recorrentes, e que foram, posteriormente, selecionadas, como ênfase da análise. A figura 3 apresenta as 20 tipologias de licenciamento mais recorrentes e seu quantitativo de requisições.

Figura 3 - Tipologias de Licenciamento Ambiental com maiores incidências de requisições

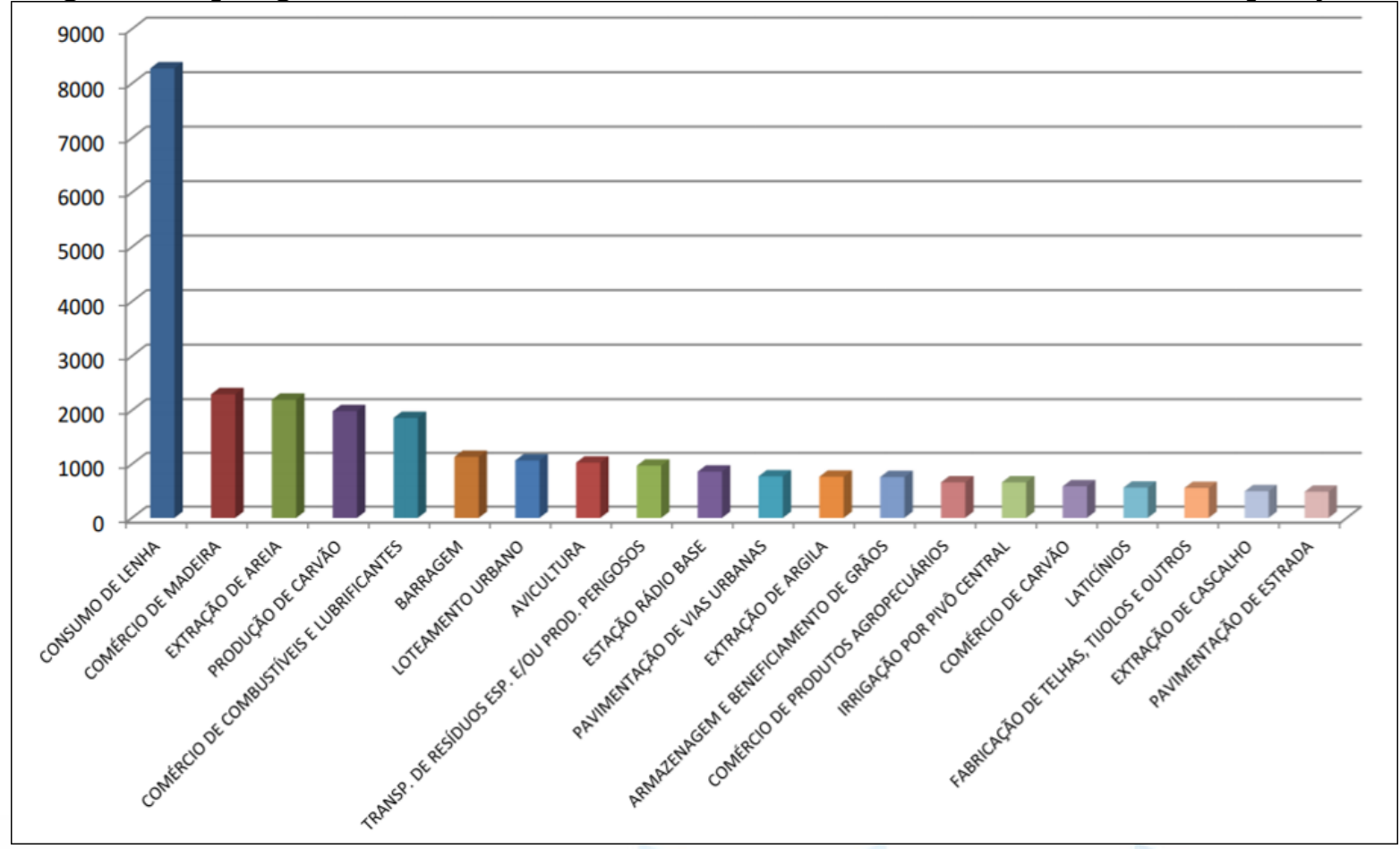

Base de dados: Sistema intranet da SECIMA (2008 a 2017). Elaborado pelos autores.

Após a identificação dos processos mais recorrentes, pôde-se avaliar e constatar quais são os critérios e instrumentos da geotecnologia que poderiam servir de apoio à análise dessas tipologias. Vale lembrar que muitos destes critérios e instrumentos sobrepõem para mais de uma tipologia e, até mesmo, para outras tipologias não ranqueadas. 
Como resposta, a avaliação revelou que a maior parcela das atividades do licenciamento ambiental não utiliza ou subutiliza os recursos mais comuns da geotecnologia. Dessa forma, é visível nas análises ambientais que definem a viabilidade dos empreendimentos, a insuficiência de instrumentos, recursos e dados relacionados à informação geográfica.

Em todas as atividades e empreendimentos verificados, foram constatadas deficiências em elementos fundamentais para a construção de um banco de dados geográfico. A maior parte dos gestores não possuem suporte para a utilização de tecnologia, realizando a análise sem o apoio de recursos fundamentais, como um acervo próprio de datas definidas de imagens de satélites, usando predominantemente o software Google Earth. Também verificou que a grande maioria dos técnicos não acessam sistemas informatizados que ofereçam dados, mesmo em escalas regionais que são informações relevantes à análise ambiental, tais como: pedologia, limites das unidades de conservação, dados de antropização do bioma, hidrografia ou qualquer outra temática do universo ambiental. Outro ponto relevante da análise foi à constatação da inexistência de um catálogo dos empreendimentos, que apresentam suporte georreferenciado.

$\mathrm{Na}$ complementação do questionário foram inseridas também, algumas questões subjetivas, além de um item em aberto para que os gestores incluíssem algumas informações não abordadas nos itens anteriores, mas que julgassem relevante para o uso da geotecnologia no licenciamento ambiental. A avaliação aponta um volume significativo de solicitações para obtenção de imagens de satélites históricas, dados referentes à hidrografia, produtos do Cadastro Ambiental Rural (CAR), Unidades de Conservação e mapeamentos do uso e cobertura da terra. Outras temáticas que se destacaram foram à malha viária e informações sobre aglomerados urbanos.

Como mecanismos de apoio foram destacados: (i) a integração dos dados cartográficos no órgão ambiental, para a geração de um banco de dados unificado; (ii) criação de um sistema interno, com ferramentas de geoprocessamento, para auxílio na análise ambiental; (iii) criação de um setor específico com profissionais habilitados em geoprocessamento; (iv) disponibilização dos empreendimentos outorgados e licenciados em um SIG online; (v) criação de subsídios de geração de produtos para GPS, com apoio de ações in loco; (vi) disponibilização na plataforma WebGIS (mapa interativo) de ferramentas que permitam a adição de polígonos, de desenho e medição, inserção de coordenadas no sistema de projeção Universal Transverso de Mercator (UTM) e Geográficas, sobreposição de layers, buffers e funções de edição dos polígonos (mover e adicionar vértices), conforme operações de análise espacial descritas por Rosa (2011) e; (vii) padronização do uso da plataforma proposta (mapa interativo) em todas as atividades de licenciamento ambiental.

Como respostas as solicitações, na página inicial da plataforma WebGIS desenvolvida, podem ser visualizados links de acesso as principais instituições de interesse. No painel de camadas, acoplado a cada feição dos mapas interativos, encontra-se um ícone para apresentação da legenda de cada temática. Essas, por sua vez, possuem separação dos itens, por lista e com simbologias distintas, para as camadas além dos ferramentais de edição. Em complementação, para o melhor gerenciamento e leitura dos dados inseridos, foi definido um catálogo resumido, que apresenta a descrição dos metadados de cada camada de informação.

A figura 4 apresenta uma das camadas do tema pedologia. Para a representação simbólica deste tema foi mantido o padrão original estabelecido, pelo órgão oficial que disponibilizou a informação. No total foram adicionados aproximadamente 200 temas relacionados ao ambiente físico e que buscavam suprir as solicitações dos gestores. Alguns temas indicados não foram localizados nas bases pesquisadas. Entretanto, aproximadamente $90 \%$ dos temas solicitados foram inseridos na plataforma. 
Figura 4 - Protótipo do WebGIS de Licenciamento Ambiental fornecido à SECIMA

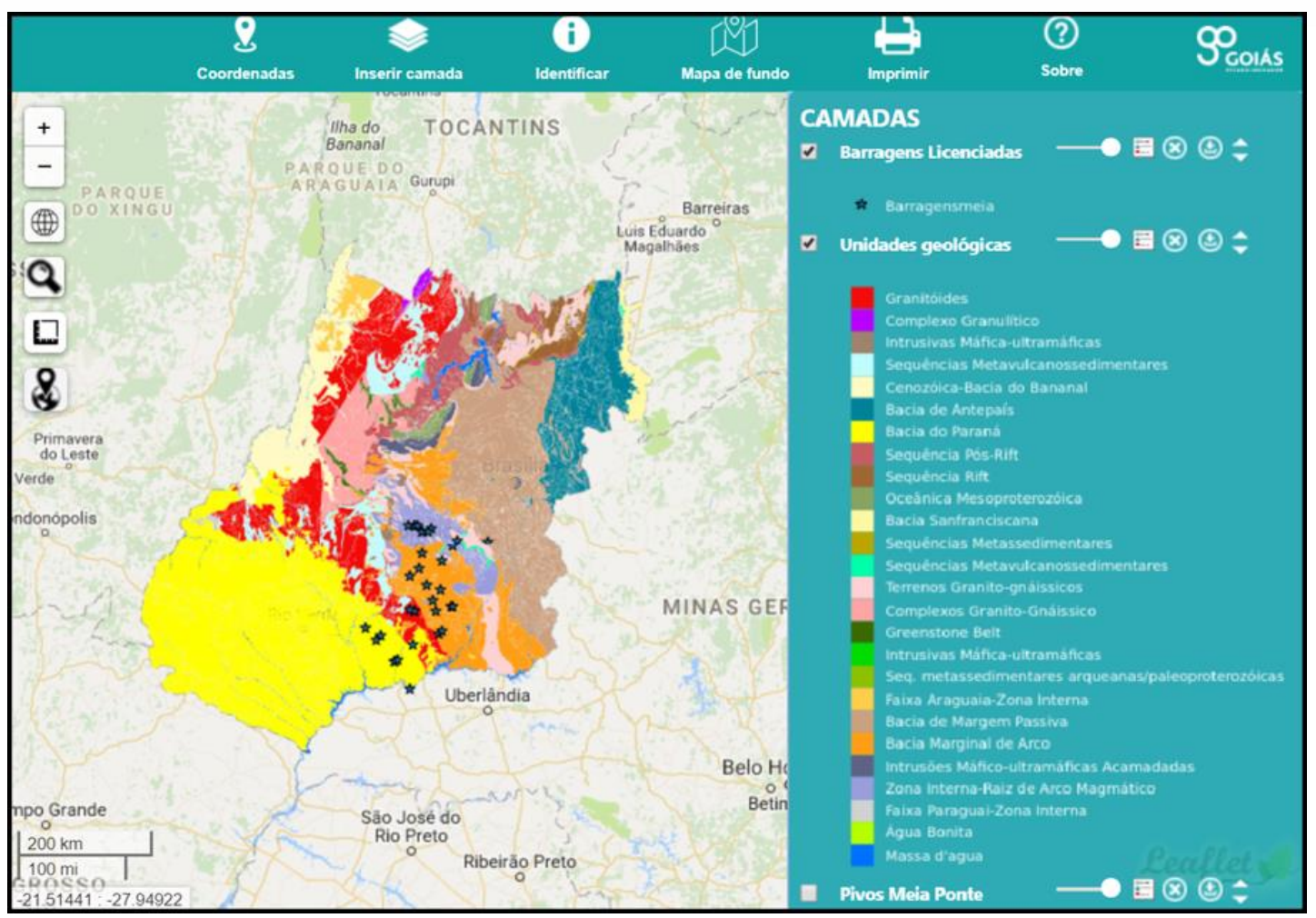

Base de dados: Superintendência de Geologia e Mineração - SIC.

Além da criação de mapas interativos para o licenciamento ambiental, foi desenvolvido um módulo para a fiscalização ambiental. Esse modelo de fiscalização, englobou três tipologias presentes no licenciamento ambiental: desmatamentos, pivôs e barragens.

Na plataforma de fiscalização, o interesse foi disponibilizar um grupo de opções para facilitar a atividade em campo. Nesta, estão disponíveis recursos para comunicação entre os agentes, balanço tabular da quantidade de processos por região e outros dados pertinentes. A arquitetura para a construção dos mapas interativos do módulo de fiscalização foi semelhante ao utilizado no do licenciamento ambiental.

Assim, os mapas interativos permitem a localização dos danos, a inserção de bases externas (open layers, imagens de satélite, topográficas, dentre outras) e a visualização de atributos tabulares que facilitem a identificação tanto de dados cadastrais, quanto do status do empreendimento/dano ambiental. Dessa forma, o fiscal ambiental poderá acessar um conjunto de informações com apenas um clique no mouse (computador com tela convencional) ou toque na tela (dispositivos móveis ou computadores com a tecnologia touch screen). Para a construção da plataforma de fiscalização foi utilizada também a codificação open-source Leaflet, da mesma forma que no mapa interativo produzido para o licenciamento ambiental que possibilita a inserção de dados vetoriais e matriciais.

\section{ANÁLISE DA NECESSIDADE DA POTENCIALIZAÇÃO DAS GEOTECNOLOGIAS}

De fato, a ausência de um setor específico de geoprocessamento e a não adoção de meios para tratamento das informações geográficas que circulam no órgão, compromete a mensuração e detecção de fatores geográficos normatizados em lei. Essa situação ocasiona consequências na emissão dos pareceres do licenciamento e desencadeia uma série de entraves 
para outras ações do órgão, comprometendo o monitoramento, a fiscalização ambiental e qualquer medida para gerir os dados espaciais.

Mesmo com a atual ausência de um setor específico de geoprocessamento, as análises feitas in loco revelaram que esse departamento pertencia ao organograma operacional da SECIMA, em gestões de governos anteriores. Obviamente, que isso não significa dizer que seu funcionamento era adequado, pois não se avaliou sua eficácia quando o departamento existia.

Entretanto, os analistas ambientais até então não dispunham de um SIG institucional que possibilitasse a obtenção de dados relacionados a fatores ambientais. Os dados fornecidos pelos responsáveis técnicos dos processos de licenciamentos, mesmo quando georreferenciados, não são inseridos em uma base de dados única, para armazenamento e gerenciamento das informações a serem compartilhadas.

Para Santos et al. (2015), essa ação revela o sentimento de propriedade sobre os dados, tão comum pelos técnicos que atuam em setores especializados e fechados, durante a obtenção e tratamento das informações espaciais. Tal situação compromete as análises futuras, não permitindo uma avaliação integrada das requisições. Assim, lembramos que no processo de licenciamento ambiental é essencial que a avaliação ocorra com multiplicidade de dados, ou seja, em uma ótica multidisciplinar (NASCIMENTO; MONTAÑO, 2011).

A falta de integração de informações compromete não apenas o licenciamento ambiental, mais também outras atividades como o Monitoramento e a Fiscalização Ambiental. O que se observa é, que o foco do licenciamento visa suprir as atividades imediatas. As informações avaliadas e geradas enfatizam a análise pontual, apoiando a equipe técnica no parecer técnico emitido para aquela determinada licença, porém, não atingindo uma visão institucional, compartilhada.

Dentre os fatores que interferem no bom funcionamento dos procedimentos de licenciamento ambiental observou-se que uma boa parcela deles, podem ser dirimidos ou reduzidos, com a adoção de padrões, técnicas e medidas convergidas a elaboração de um SIG para avaliar, gerenciar e dimensionar as ações com reflexos ambientais.

Dessa forma uma mudança de paradigma, envolveria não somente a aquisição de um $S I G$ institucional e a obtenção de uma base de dados mais robusta, mas também a compilação de informações que busquem ações fortalecedoras da análise e fiscalização ambiental. Neste entendimento, a percepção das potencialidades das geotecnologias para aquisição de dados e do SIG para manipulação destes, por parte dos agentes ambientais é de extrema importância para implantar propostas, tais como a sugerida neste artigo.

Nesse aspecto, o questionário aplicado demonstrou que os gestores que participaram da análise são unânimes na importância do uso da geoinformação. Também apontaram que a plataforma apresentada, pode contribuir, significativamente para a avaliação mais detalhada dos processos ambientais. Essa percepção da relevância da adoção de meios de melhor acessar, gerenciar e disseminar as informações geográficas é um fator crucial para a implementação do sistema e demais ações decorrentes.

Até então, o analista ambiental normalmente efetuava uma análise pontual, desconsiderando a sinergia entre o meio ambiente e os empreendimentos anteriormente cadastrados. Ademais, a análise do volume de processos licenciados, obtida via sistema intranet do órgão ambiental, demonstrou outro importante aspecto do licenciamento ambiental no Estado de Goiás. Diversas tipologias apresentaram um quantitativo de licenças muito inferior ao volume de dados registrados, mapeados e verificados por outras instituições. Como exemplo, foi constatada uma considerável discrepância entre a quantidade de licenças emitidas no período consultado e a quantidade de pivôs e barragens presentes no arquivo do repositório do SIEG, proveniente de mapeamentos efetuados pela Secretária da Fazenda de Goiás (SEFAZ).

Como ilustração, a figura 5, apresenta uma área no município de Jussara/Goiás. O levantamento de licenças entre 2008 e início de 2017, no município indica que foi emitida pela SECIMA apenas uma (1) licença para a tipologia de pivô de irrigação central. Somente na área apresentada na figura citada, que contempla parte do território de Jussara, nota-se um volume 
substancialmente superior de pivôs centrais. Outro exemplo dessa situação é no município de Cristalina. O levantamento da Secretaria da Fazenda em 2013 aponta 659 pivôs centrais no município. Para as barragens, o mapeamento efetuado pelo Instituto Mauro Borges (IMB), identificou 328 barragens, enquanto foram licenciadas entre 2008 até janeiro de 2017 apenas nove (9), empreendimentos no município.

Figura 5 - Imagem do aplicativo de fiscalização demonstrando área de pivôs centrais no município de Jussara/GO

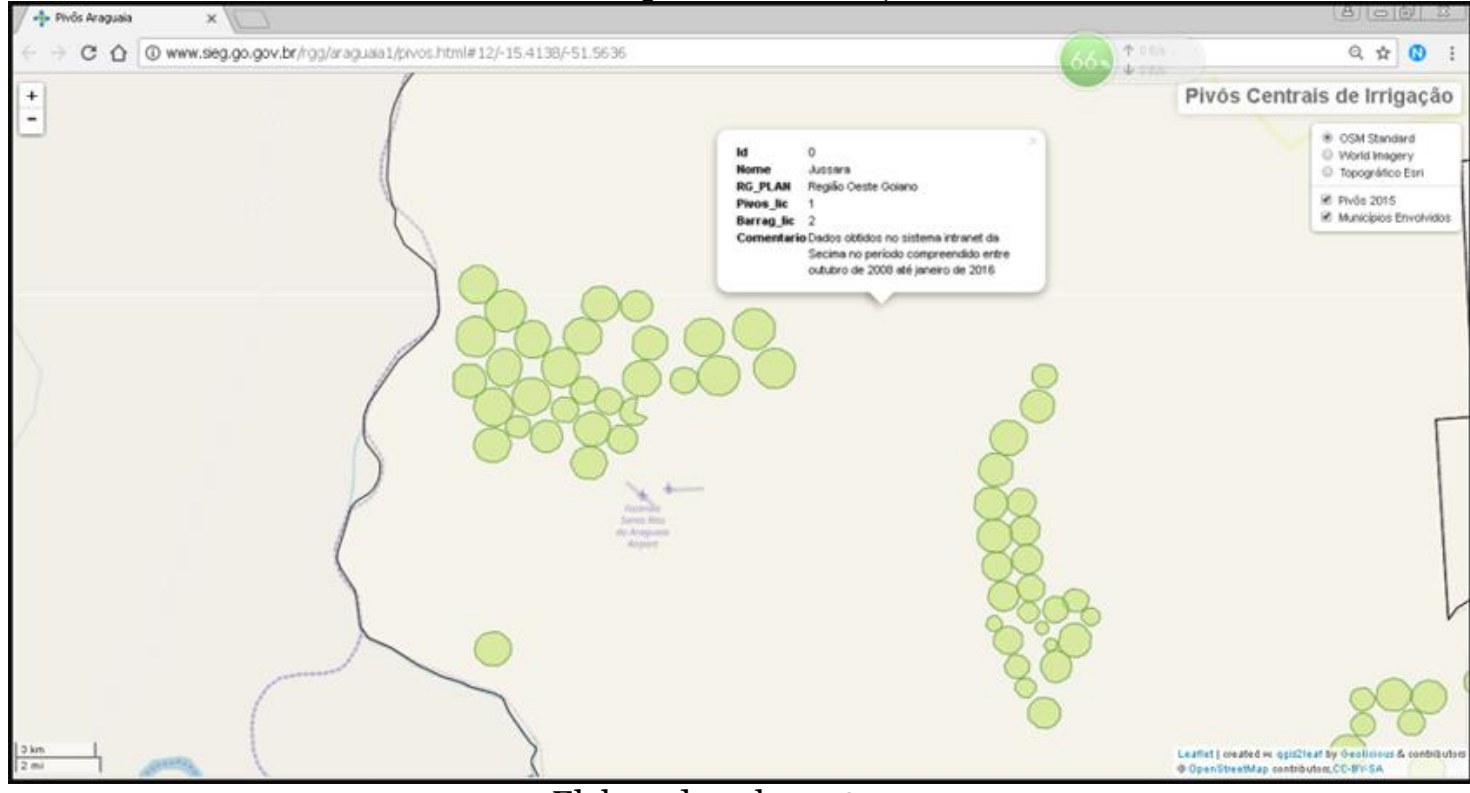

Elaborado pelos autores.

Tal circunstância estimula a ponderação para a construção de um novo paradigma no licenciamento ambiental goiano. Determinadas tipologias possuem um volume de empreendimentos irregulares que superam os empreendimentos regulamentados. Cabe salientar, a prudência de analisarmos se efetivamente o órgão ambiental centraliza suas ações de gestão de forma ordenada em determinados cenários. Tal situação pode ser reforçada por Hofmann (2015), que aponta como um dos gargalos do licenciamento ambiental a ausência de dados e de informações ambientais sistematizadas, além do excesso de burocracias.

É visível que em uma parcela significativa dos processos de licenciamento o fator geográfico não é devidamente dimensionado. Essa inadequação consiste em um dos principais obstáculos do licenciamento, haja vista, que algumas atividades ou empreendimentos não contabilizam toda a influência decorrente da implantação de sua obra no espaço geográfico. Nesses termos, a ausência de um gerenciamento espacial, acarreta limitações na alocação posicional dos empreendimentos, em virtude tanto do desconhecimento de dados para a implantação de um empreendimento (aspecto pontual), quanto na ausência de instrumentos que computem os impactos sinérgicos e cumulativos, dos outros empreendimentos, instalados no entorno (ausência de integração).

Para amenizar essa situação, a institucionalização do registro posicional de todos os empreendimentos, associada a padronização para fornecimento de produtos cartográficos revela-se como uma oportunidade de catalogar, registrar e localizar os empreendimentos licenciados. Para a consecução dessa iniciativa, a adoção do SIG, em conjunto com a criação de normatizações técnicas, estabelecendo regras para os produtos cartográficos fornecidos pelos responsáveis técnicos, permitirá uma melhor aplicação de um banco de dados geográfico mais robusto.

Cabe destacar ainda que a padronização dos dados cartográficos auxiliará tanto na gestão ambiental, quanto nas próprias rotinas administrativas de análise de processos. Com a

Helber de Carvalho, Fabrizia G. Nunes, Alex M. dos Santos. Sistema de informação geográfica como ferramenta de apoio ao (...) Brazilian Geographical Journal: Geosciences and Humanities research medium, 
implantação de um SIG, em determinados cenários será possível reduzir a subjetividade e o aspecto isolado da análise, sobretudo quando se tratar de análises quantitativas e direcionadas pela lei.

Entretanto, dentre os aspectos que ainda devem ser revistos nas legislações ambientais, destaca-se a sobreposição e conflito de competências decorrentes da participação dos três entes federativos no processo de licenciamento. Nesse contexto é essencial um melhor dimensionamento e delineamento da competência de cada esfera participante do processo. Outro ponto fundamental é a elaboração de normas objetivas no licenciamento ambiental, assegurando que as análises ocorram em um contexto mais imparcial, sem o questionamento, motivado por algum agente específico. Isso impede que o resultado do licenciamento fique dependente de qual será o técnico que realizará a análise.

A pesquisa revelou ainda que é reduzido o número de servidores em Goiás que atuam diretamente com o Licenciamento Ambiental e que têm habilitação específica, em geoprocessamento e/ou treinamento para utilizar dados geoespaciais. Isso compromete a obtenção de diversas potencialidades para as inúmeras atividades que envolvem a informação geográfica e impede o adequado uso dos softwares de SIG. A não consideração plena da variável locacional como um dos parâmetros para classificar e direcionar o licenciamento ambiental compromete a correta adoção de instrumentos como o Zoneamento EcológicoEconômico, planos de gestão de bacias hidrográficas, Unidades de Conservação, espécies protegidas, dentre outros (HOFMANN,2015).

Goiás possui instituições que mantém importantes repositórios para acesso de informações geográficas. Entretanto, apesar de constituir fontes de consulta para as ações do licenciamento ambiental, existe a limitação para a customização de ferramentas e integração com dados específicos do licenciamento. É preciso um sistema que disponibilize todos os dados integrados e demandados no ato do licenciamento ambiental, além de mecanismos que incorporem o aspecto posicional de todos os empreendimentos e atividades requisitantes de licenciamento.

De todos elementos citados pelos gestores entrevistados, consideramos como o maior desafio, a disponibilização dos empreendimentos outorgados e licenciados em um ambiente de SIG online/WebGIS. Nesse cenário, os usuários (analista/técnico e/ou gestor ambiental) teriam acesso ao perímetro ou coordenada central do empreendimento, permitindo além da visualização do licenciamento em análise, visualizar os demais licenciamentos que foram incluídos nas solicitações, além de todas as camadas compiladas nos repositórios de dados.

Para atender este desafio, é importante uma atuação multifacetada, sendo necessária a mobilização de diversos setores do órgão ambiental para adequar suas ações na padronização dos dados cartográficos. Isso contempla desde a adequação da informação entregue nas requisições de licenciamento ambiental e no oferecimento de cursos para os profissionais envolvidos, até a garantia de meios para validar e incluir automaticamente as informações em um banco de dados geográfico.

Nas reuniões com os gestores ambientais, foi apresentada a potencialidade da utilização de uma base de dados integrada. Nesse momento foi salientado que a própria natureza da análise ambiental necessita de avaliações sistêmicas. Sendo que a integração das informações é o passo inicial para uma avaliação mais consistente, das análises ambientais, processos de monitoramento e fiscalização ambiental.

Dessa forma, a apresentação do protótipo enfatizou a utilização de instrumentos das geotecnologias que agreguem funções destinadas a um sistema de licenciamento. O usuário poderá acessar centenas de dados georreferenciados de fontes oficiais, além da capacidade de assimilar dados provenientes dos estudos ambientais presentes nas requisições de licenciamento. Após acessar o módulo de visualização, o usuário visualiza os dados com uma interface Leaflet, uma aplicação gratuita que possui ferramentas diversificadas que podem otimizar a compreensão dos dados inseridos.

A grande maioria dos técnicos ainda utilizam para a análise mapas impressos ou nos formatos "pdf" ou "jpg" para efetuar a análise. Assim, a disponibilização dessa ferramenta com

Helber de Carvalho, Fabrizia G. Nunes, Alex M. dos Santos. Sistema de informação geográfica como ferramenta de apoio ao (...) Brazilian Geographical Journal: Geosciences and Humanities research medium, Ituiutaba, v. 11, n. 2, p. 4-21, ago./dez. 2020 
interface amigável e com um volume substancialmente maior de informações fornecerá uma nova perspectiva na análise ambiental.

No que concerne à produção de dados, sugere-se que em trabalhos futuros além das etapas de seleção, compilação, edição dos dados, também ocorra o refinamento de dados. Essa atuação é bastante necessária porque, uma boa parcela dos dados atuais disponíveis, apresentam escalas incompatíveis com uma análise refinada. Um exemplo é o mapa de solos do Estado Goiás, disponível no site do SIEG, com escala 1:1.000.000. Essa escala permite uma avaliação sob o escopo estadual, mas não é compatível com as análises do Licenciamento Ambiental, que tratam em muitas circunstâncias de propriedades rurais. Aqui, é importante frisar que instrumentos computacionais que forneçam informações geográficas e descritivas sobre temas vinculados aos recursos ambientais, acarretam agilidade e eficiência a diversos processos de gestão ambiental, principalmente aos relacionados com licenciamento ambiental (VENDRUSCO et al., 2009).

\section{CONSIDERAÇÕES FINAIS}

No decorrer deste trabalho foram identificadas dificuldades e limitações nos procedimentos do Licenciamento Ambiental. Quanto as dificuldades, observou-se que os dados e as informações geográficas utilizadas pela SECIMA não são armazenadas com o mesmo rigor cartográfico, em uma base de dados unificada e compartilhada. Parcela considerável de seus entraves é decorrente da inexistência de uma Base Cartográfica robusta e eficaz, e que atenda, todas as demandas das análises. Portanto, a estruturação do WebGIS institucional foi dificultada.

Com a pesquisa pode verificar também que durante a avaliação dos empreendimentos, os gestores ambientais até o presente, não usufruíam de um WebGIS que permitisse a obtenção de dados relacionados a fatores ambientais. Os dados fornecidos pelos responsáveis técnicos dos processos de licenciamentos, mesmo quando georreferenciados, não são inseridos em uma base para armazenamento e gerenciamento. Além disso, como já mencionado, não são todos os técnicos da SECIMA que possuem habilitação específica em geoprocessamento e/ou treinamento para utilizar dados geoespaciais. Essas limitações estão presentes em todas as fases de tramitação do processo ambiental. Isso envolve desde a apresentação dos documentos cartográficos, que compõem o processo, até o momento da emissão da licencia

Nesse contexto, iniciativas de instrumentalização computacionais, como o WebGIS apresentado neste artigo poderá fornecer informações geográficas e descritivas sobre uma diversidade de temas vinculados aos recursos ambientais, acarretando na agilidade e eficiência de diversos processos de gestão ambiental, principalmente naqueles relacionados com o Licenciamento Ambiental do órgão ao qual foi destinado. Desta forma, o desenvolvimento deste trabalho buscou gerar, a partir da convergência das pesquisas bibliográficas e da averiguação do panorama atual da SECIMA, uma proposta de Mapa Interativo (WebGIS), aplicado ao atual cenário do Licenciamento Ambiental do Estado de Goiás. Como resposta, almeja-se que a adoção dos métodos e ferramentais computacionais propostos, colaborarem no direcionamento de estratégias para tomada de decisão, atendendo as demandas governamentais com maior velocidade e eficiência, ocasionando uma avaliação ambiental pautada nos aspectos legais e sustentáveis.

Além da apresentação do protótipo do WebGIS foi oferecida também uma formação e avaliação de seu desempenho junto aos participantes, que são os técnicos da SECIMA. Contudo, é importante salientar que esta formação deve ser continuada, para atingir igualitariamente todos dentro de suas limitações. Como perspectiva de trabalhos futuros, sugerese que além da compilação de dados provenientes de diversas fontes, que sejam produzidos novos dados para uma melhor constituição da Base Cartográfica, e que contenha um repositório com mais diversidade de informações e com escalas mais apuradas. 


\section{AGRADECIMENTOS}

Os autores agradecem ao pronto atendimento no fornecimento de dados da Secretaria do Meio Ambiente, Recursos Hídricos, Infraestrutura, Cidades e Assuntos Metropolitanos (SECIMA). Outra instituição fundamental na pesquisa foi o Sistema Estadual de Geoinformação de Goiás (SIEG), representado pelo Instituto Mauro Borges de Estatísticas e Estudos Socioeconômico IMB, parceiro da SECIMA.

\section{REFERÊNCIAS}

BARRETO, Flávia R. de Mattos. Análise da etapa de delimitação do escopo em processos de Avaliação de Impacto Ambiental. 2012. 230f. Mestrado em Ciências da Engenharia Ambiental - Curso de pós-Graduação em Ciências da Engenharia Ambiental de São Carlos/USP. São Carlos.

BRASIL. Conselho Nacional de Meio Ambiente. Resolução CONAMA n. ${ }^{\circ}$ oo1. Dispõe sobre critérios básicos e diretrizes gerais para a avaliação de impacto ambiental. Brasília: 1986. Diário Oficial da União. 17 de fevereiro de 1986.

BRASIL. Conselho Nacional de Meio Ambiente. Resolução CONAMA n. ${ }^{\circ}$ 237. Dispõe sobre a revisão e complementação dos procedimentos e critérios utilizados para o licenciamento ambiental. Brasília: 1997. Diário Oficial da União. 22 de dezembro de 1997.

BRASIL. Lei n. ${ }^{\circ} 6.938$ de agosto de 1981. Dispõe sobre a Política Nacional do Meio Ambiente, seus fins e mecanismos de formulação e aplicação, e dá outras providências. Brasília: 1981.

CÂMARA, G.; DAVIS, C. Arquitetura de Sistemas de Informação Geográfica. In: Introdução à ciência da Geoinformação. São José dos Campos: Divisão de Processamento de Imagens (DPI) e Instituto Nacional de Pesquisas Espaciais (Inpe), 2004. Disponível em: <http:www.dpi.inpe.br/gilberto/livro/introd/>. Acesso em: 28 jan. 2019.

CÂMARA, G.; MONTEIRO, A. M.; MEDEIROS. J. S. Fundamentos epistemológicos da Ciência da Geoinformação. In: Introdução à ciência da Geoinformação. Livro on-line, INPE. 2000. Disponível em: <http://www.dpi.inpe.br/gilberto/livro/introd/cap5epistemologia.pdf $>$. Acesso em 15 ago. 2019.

CÂMARA, G.; MEDEIROS, J. S. de. Geoprocessamento para projetos ambientais. 1. ed. São José dos Campos: Instituto Nacional de Pesquisas Espaciais (Inpe). 1998.

CARVALHO, H.; MITIDIERE, M. V.; OLIVEIRA, W. N. Uso de geotecnologias para monitoramento e fiscalização do desmatamento no estado de Goiás: uma avaliação associada ao licenciamento ambiental. In: XVI Simpósio Brasileiro de Sensoriamento Remoto, 2013, Foz do Iguaçu. Anais do XVI Simpósio Brasileiro de Sensoriamento Remoto. Foz do Iguaçu: INPE, 2013, p. 4459-4466.

DA ROCHA, D. S. Potencialidades do uso de um sistema WEBGIS na pecuária de precisão: estudo de caso. 1. ed. Santa Maria: Embrapa Pecuária Sul, 2014.

ESTES, J.; STAR, J. Geographic Information Systems: an introduction. 1. ed. Santa Bárbara: Universidade da Califórnia, 1990.

FAVRIN, Vanessa Garcia. As geotecnologias como instrumento de gestão territorial integrada e participativa. 2009. 231f. Mestrado em Geografia Humana - Faculdade de Filosofia, Letras e Ciências Humanas, Universidade de São Paulo, São Paulo. 
HOFMANN, R. M. Gargalos do licenciamento ambiental federal no Brasil. Consultoria Legislativa: Câmara dos Deputados, Brasília - DF, 2015.

MELCHIORS, J. L.; MEDEIROS, R. M. V. O processo histórico dos territórios agrários de Eldorado do Sul-RS: a entrevista semiestruturada e a observação como técnicas de pesquisa qualitativa em Geografia. In: PESSÔA, V. L.; RUCKERT, A. A.; RAMIRES, J. C. L. (Org.). Pesquisa qualitativa: aplicações em Geografia. Porto Alegre: Imprensa Livre, p. 462-487, 2017 (livro digital).

NASCIMENTO, L. A.; MONTAÑO, M. Desenvolvimento de um modelo aplicado de Sistema de Informação Ambiental para suporte ao licenciamento ambiental no estado de São Paulo. In: XV Simpósio Brasileiro de Sensoriamento Remoto, 2011, Curitiba. Anais do XV Simpósio Brasileiro de Sensoriamento Remoto. Curitiba: INPE, 2011. v. 1. p. 8819-8826.

OLIVEIRA, P. T. S. de; AYRES, F. B.; PEIXOTO FILHO, G. E. C. da; MARTINS, I. P. MACHADO, N. M. Geoprocessamento como ferramenta no licenciamento ambiental de postos de combustíveis. Revista Sociedade \& Natureza, Uberlândia, v. 20, n.1, p. 87-99, 2008.

PAES, Hélio Garcia. Contribuições da geografia ao licenciamento e ao estudo de Impacto Ambiental. 2010, f. 149. Mestrado em Geografia - Pós-Graduação em Geografia Física da FFLCH/USP, São Paulo.

ROSA, R. Análise Espacial em Geografia. Revista da ANPEGE, v. 7, n. 1, número especial, p. 275-289, 2011.

SAITO, Estela Rosa Federmann. Cenário do licenciamento ambiental: principais entraves-análise das competências ambientais no sistema federativo brasileiro. 2010, f. 157. Mestrado em Direito - Programa de Pós-Graduação em Direito da PUC/SP, São Paulo.

SÁNCHEZ, L. E. Avaliação de Impacto Ambiental: conceitos e métodos. 1. ed. São Paulo: Oficina de Textos, 2013.

SANTOS, A.M.; BUENO, L.F.; SAMPAIO, T.V.M. Dados e informações geoespaciais para análise territorial e ambiental na Amazônia Legal no Brasil. Revista Geográfica Venezolana. Venezuela, v. 56, n .2, p. 249-267, 2015.

SPERANZA, E. A.; SILVA, J. S. V. da. Ferramenta computacional para auxílio à análise técnica de processos de licenciamento ambiental utilizando geotecnologias. In: $3^{\mathrm{o}}$ Simpósio de Geotecnologias no Pantanal, 2010, Cáceres - MT. Anais do $3^{\mathbf{o}}$ Simpósio de Geotecnologias no Pantanal. Cáceres: Embrapa Informática Agropecuária/INPE, 201. p. $867-876$.

TANG, F. et al. A Web GIS Platform for Environmental Livelihood Value Assessment in Northeastern British Columbia. Spatial Knowledge and Information Canada, v. 7(1), n.3, p. 1-7, 2019.

VATRESIA, A.; SADLER, J. P.; RAIS, R. R. SUSTINERE. Journal of Environment and Sustainability. v. 1, n. 1, p. 10-22, 2017.

VENDRUSCULO, L. G., SILVA, J., ARAÚJO, L. B., OLIVEIRA, S. D. M., \& COPATTI, A. Uso da técnica de Web Mapping para disseminação de dados ambientais com enfoque no licenciamento ambiental). In: Simposium Iberoamericano en Generación, Comunicación y Gerencia del Conocimiento; Conferencia Ibero-Americana en Ingeniería e Innovación Tecnológica, 2009, Orlando. Anais do Simposium International Institute of Informatics and Systemics. Florida: Memorias Embrapa Informática Agropecuária(ALICE), 2009. 
ZHENG, Z; CHANG, Z. Y.; FEI, Y. F. A simulation-as-a-service framework facilitating WebGIS based installation planning. The International Archives of the Photogrammetry, Remote Sensing and Spatial Information Sciences, v. XLII-2/W7, ISPRS Geospatial Week, p. 18-22, 2017.

Recebido em: 05/04/2020.

Aprovado para publicação em: 03/11/2020.

Helber de Carvalho, Fabrizia G. Nunes, Alex M. dos Santos. Sistema de informação geográfica como ferramenta de apoio ao (...) Brazilian Geographical Journal: Geosciences and Humanities research medium, Ituiutaba, v. 11, n. 2, p. 4-21, ago./dez. 2020. 